Copyright (C) 201X Inderscience Enterprises Ltd. 


\title{
Supporting Collaborative Business Processes: a BPaaS Approach
}

\section{Lai Xu}

Department of Computing and Informatics, Faculty of Science and Technology, Bournemouth University, Poole, UK

E-mail: lxu@bournemouth.ac.uk

\section{Paul de Vrieze}

Department of Computing and Informatics, Faculty of Science and Technology,

Bournemouth University,

Poole, UK

E-mail: pdvrieze@bournemouth.ac.uk

\begin{abstract}
Collaborative business processes are increasingly driven by business flexibility and agility. Cloud-based business process management services have provided small medium enterprises (SMEs) with a pay-per-use manner for their daily business needs, i.e. some simple business process applications, e.g. salesforce provides cloud-based CRM to boost SMEs' sales. This raises the question how cloud-based business process management solutions can support the fast pace of change of business collaborations among business partners? For example, collaborative processes for managing industrial incidents are short term, low frequency processes. This paper proposes an architecture meta-model, which is used to design the concrete architecture and to further analyse the performance of the proposed solution. A real world case of collaborative processes for incident and maintenance notifications is used to explain the design and implementation of the cloudbased solution for supporting collaborative business processes. Service improvement of the new solution and computing power costs are analysed accordingly.
\end{abstract}

Keywords: Business Process as a Service (BPaaS); Incident Management; Business Process Mashup; Cloud Computing; Service-oriented Computing

Biographical notes: Dr. Lai Xu is a senior lecturer in faculty of Science and Technology (SciTech) of Bournemouth University. Previously she was a Senior Researcher at SAP research, Switzerland and a Senior Research Scientist at CSIRO ICT Centre, Australia. She received her Ph.D. in Computerized Information Systems from Tilburg University, the Netherlands in 2004. Dr. Xu is a coordinator of the EU H2020 FIRST project. Her main research interests include smart technologies, virtual factory, digital factory, smart factory, business process management, service oriented computing, big data and cloud computing, business process as a service (BPaaS), process modelling and simulation, process mashups, business process collaboration, and business process modelling, interoperability, trust and security issues in process collaborations.

Dr. Paul de Vrieze is a senior lecturer faculty of Science and Technology (SciTech) of Bournemouth University. Previously he was a Senior Researcher at SAP research, Switzerland, and worked as a Postdoctoral Research Fellow at CSIRO ICT Centre, Australia. He received his Ph.D. in Information Systems from Radboud University Nijmegen, The Netherlands in 2006. He is a co-coordinator of the EU H2020 FIRST project. His main research interests are in enterprise information system integration, user modeling, systems modeling, collaboration process modelling and simulation, semantics integration and tracability of process collaborations.

\section{Introduction}

Cloud computing provides distributed infrastructure and elastic computing power for the needs of organisations of different sizes. There exist a number of different types cloud-based business process (BP) services for a single organisation, namely IaaS BP services, PaaS $\mathrm{BP}$ services, and SaaS BP services. In collaborative organisations cloud computing is a good way to provide interconnection for the participants. In this context, cloud based business process management can also be an ideal solution for collaborative business process management; cloud-based solutions provide on-demand 
services and resources in a distributed way to support flexbility and agility of collaborative business processes.

From an overall perspective industrial incidents are inevitable. Some of them relate to our everyday life and may have strong adverse impacts on our society and economy. Managing incidents generally involves multiple parties/organisations. The aim of incident management is to reduce the impact of the incidents and it requires joint efforts from all involved stakeholders. The process of handling incidents is a typical collaborative process with urgency and short duration of use.

There are different solutions for supporting collaborative processes across multiple organisations, such as setting e-contracts among involved partners' systems (Xu and Jeusfeld, 2003), (Kutvonen et al., 2005), (Kutvonen et al., 2007) as well as service orchestration and choreography among different organisations (Leymann et al., 2002; Xu, 2011). The above mentioned solutions are however designed to support long-term, fixed, trusted collaborative processes where the value of repeatability and reliability is high.

A consequence of this is that in practice, many short term, low frequency processes are not computer supported due to implementation complexity. In a collaborative context this complexity is even more relevant. This implementation complexity of collaborative business process automation depends on the flexibility of the process to be automated, the cardinality of participating business processes and the correlation of collaborating process instances (Xu et al., 2013a).

In this paper, we provide a cloud-based business process solution for these fast changing collaborative business processes, i.e. a cloud-based collaborative business process solution for multiple organisations involved in incident notification processes. Main contributions of this research are threefold:

- An architecture meta-model; specified to support collaborative business processes. The architecture meta-model is used to design a concrete architecture and to further analyse the performance of the proposed solutions.

- An incident and maintenance notification case shows the agility of collaborative process. The case is used to explain the design and implementation of BPaaS for supporting collaborative business processes.

- A simulation based analysis of service improvement and computing power costs of the provided solution.

\subsection{Background for Cloud-based Business Process Management}

The Internet is the predominant conduit for information exchange. In this role it has fundamentally changed how business is conducted, improving connectivity and availability at very low cost. Cloud computing allows the use of computing resources in a pay-per-use manner as well as regarding these resources as elastic (Bouvry, 2014; Duipmans et al., 2014).

In the cloud computing age, business process management systems have also adapted themselves to cloud environments. Different concepts related to business process management systems have appeared from various sources, i.e. Business Process as a Service (BPaaS), Business Process Management as a Service (BPMaaS), workflows in cloud, etc .

Even for the concept of Business Processes as a Service (BPaaS) there are different definitions. Accorsi (2011) defines the BPaaS Cloud computing model as a special SaaS provision model in which enterprise could offerings provide models for the modelling, utilization, customization, and (distributed) execution of business processes. The earlier work of Papazoglou and van den Heuvel (2011) corresponds to this BPaaS definition by Accorsi. This approach to $\mathrm{BPaaS}$ emphasizes a focus on enterprise specific services. It lets developers create unique end-to-end business processes that are usually syndicated with other external services (possibly provided by diverse SaaS providers). This BPaaS approach requires effective management of cloud resources (Papazoglou and van den Heuvel, 2011). Cloud syndications at the SaaS level are termed BPaaS (Bentounsi et al., 2016). Abovementioned BPaaS definitions view BPaaS as a special SaaS model.

There are, however, later BPaaS definitions which have a view of BPaaS beyond BPaaS as only a special SaaS model. Woitsch and Utz (2015) define BPaaS as a concept where models and semantics are applied to align business processes with cloud deployed workflows. In this approach BPaaS (Zhang and Zhou, 2009) can be any type of business process to be delivered through cloud infrastructure (Xu et al., 2013a).

Further cloud-based business process applications and implementations related to BPaaS concepts can be identified from (Han et al., 2010; Santos and Pires, 2012; Duipmans et al., 2014), etc. These cloud-based business process applications are implemented across the SaaS paradigm.

The perspective of BPaaS used in this paper is thus based on this perspective to mean that BPaaS is a concept in models and semantics are applied to align business processes with cloud deployed business processes and business process management. Based on a cloud delivery model, BPaaS usage has further been classified as SaaS based Business Process Service (SaaS BP Service), PaaS based Business Process Service (PaaS BP Service), and IaaS based Business Process Service (IaaS BP Service). As workflows are specific business processes (Van Der Aalst et al., 2003), workflows in the cloud can thus be seen as a kind of BPaaS.

SaaS BP Services provide process-oriented capabilities as ready-made solutions for smaller organisations or specific business functions or departments of big organisations. An example of this 
is the cloud-based Salesforce CRM. SaaS BP services provide users a cloud-based BP solution as a preconfigured system where all business processes are predefined or only slightly modified based upon simple configuration variables (Xu et al., 2013a). A significant advantage of using SaaS based BP services is that it forgoes the need of specialist administration as well as up-front investments and later maintenance in software and hardware.

When existing BPM systems migrate to cloud-based platform services, it is seen as PaaS BP services. PaaS $\mathrm{BP}$ is effectively defined as the provision of process execution as a platform for custom processes. The engines run in the cloud and execute different business processes for different users or organisations. BPMaaS can thus be viewed as a special PaaS BP service model of BPaaS that fits particularly well with migration of existing processes.

Individual users or organisations specify their business logic and process models according to their individual needs. These business process models are then executed by the business process engine in the cloud. Different users i.e. organisations are not sharing business process models, instead the PaaS cloud providers offer a reliable business process management platform, business process execution, data storage capabilities, etc. PaaS $\mathrm{BP}$ services save the costs of expensive hardware and BPM software, as well as the maintenance cost, e.g. the cost of updating software. Multiple organisations share same business process management platforms and each of them is responsible for its business process design, process models, and data.

It is also possible to migrate existing BPM systems onto cloud infrastructure, i.e. a new hardware environment, which can be seen as IaaS based BP services. The only change for the organisation is where the BP systems runs. The organisation owns its software, such as the business process management system and other artefacts, for example, business process logics/models, process activities, and data. VPN (Virtual Private Network) connections with the provider can make the internal systems available. The advantage is that the organisation does not need to do maintenance and/or to update the hardware, which is taken care of by the infrastructure cloud provider.

From a general cloud classification perspective, the above mentioned cloud-based BP services, i.e. SaaS BP services, PaaS BP services, IaaS BP services, are generally classified as cloud-based BP services, i.e. BPaaS. These general views focus on providing on-demand platforms (i.e. PaaS BP services) and software over the Internet (i.e. SaaS BP services) in addition to selling traditional software licenses for server installations. Elastic Business Process Management (EBPM) (Stefan et al., 2015) recently emerges a new trend to execute processes on resource elasticity of a cloudenabled BP system. E-BPM does not conclusively belong in any of the groups of SaaS BP services, PaaS BP services, or IaaS BP Services. It can be configured such that it is a SaaS BP service, PaaS BP service, or IaaS BP Service or combination thereof. However, E-BPM is also viewed as a kind of BPaaS.

\subsection{Collaborative Business Process Management}

Collaborative business processes exist not only in a single company between different departments/divisions, but also among different organisations. These interorganization processes can strongly benefit from cloud based BPM. To determine the best ways to do so it is important to review the different technologies and approaches that support collaborative business processes.

A review by Niehaves and Plattfaut (2011) finds that most cross-organizational collaborative business processes can be found from global value chains(Gereffi et al., 2005) (Sia et al., 2008), production networks(Sturgeon, 2002), interconnected firm (Lavie, 2006), collaboration dynamics(Afuah, 2001), outsourcing(Katila and Mang, 2003), etc. Research fields such as business process management, workflow systems, and computerized information systems, are considering design, deployment and implementation related information systems to support above mentioned applications.

Contract or agreement based collaboration approaches have been broadly used to support virtual enterprises (Gou et al., 2003)(Xu et al., 2010), supply chains (Muckstadt et al., 2001), inter-organisations (Norta and Grefen, 2007), and e-commerce (Xu and Jeusfeld, 2003), (Kutvonen et al., 2005), (Kutvonen et al., 2007). Collaboration processes can be also supported by service orchestration and choreography (Leymann et al., 2002), (Xu, 2011) among different organisations.

The prevalence of collaborative activities among organizations means that their management is a significant issue. $\mathrm{Mu}$ et al. (2011) provide a modeldriven BPM approach for designing a Mediation Information System. Such a collaborative BPM approach is classically dedicated to design process cartography for three types of goals: (i) certify the modelled organization, (ii) optimize the processes of the organization, and (iii) define the requirements for the information system design of the organization.

Other work has explored additional support for collaborative processes execution and management. Blanc et al. (2007) describe evolution management of supply chains. Norta and Grefen (2007) discover patterns for inter-organizational business process collaboration. Verification of collaborative processes' deadlock-freeness is discussed in by Klai et al. (2011). Collaborative process modelling related issues are discussed by zur Muehlen et al. (2005), Liu and Shen (2003), Xu et al. (2013a), and Xu et al. (2010).

The existing process modelling techniques provide different business process capabilities. Various process modelling techniques have a graphical notation, e.g. 
BPMN (OMG, 2006), Workflow Nets (van der Aalst, 1996), YAWL (Van Der Aalst et al., 2004), EPC (Keller et al., 2009) etc. Other business process modelling techniques, generally not graphical, support declarative business process modelling, which allows specifying processes through a formalism (generally Linear Temporal Logics (LTL)) meaning that the control flow is implicitly defined by the constraints of the processes, e.g. DECLARE (Pesic et al., 2007).

Business artifacts (Nigam and Caswell, 2003) allow for the definion of an operational model of business processes in which the changes and evolution of business data, or business entities are considered as the main driver of the processes. Although this and other artefactcentric business processes are founded on a wellestablished theory, the level of technological support is still not sufficient. The technical challenges to support cloud-based artifact-centric business processes has been extensively explored by Sun et al. (2014), Sun et al. (2016), and Yu et al. (2015).

The main difference between workflow based business processes and artefact-centric business processes is how to treat the data involved in process. In workflow based business process systems data is not seen as the driver of the process. Instead, workflow based business processes are centered around the control flow. The well-known workflow control-flow patterns (Russell et al., 2006) characterise the range of control flow constructs that might be encountered when modelling and analysing workflows. The work also considers data patterns (Russell et al., 2005) aiming to capture the various ways in which data is represented and utilised in workflows.

Based on the well-established base of workflow based business processes and the technical challenges of artefact-centric business process management this paper addresses the problem from the workflow perspective as set out in the workflow patterns work. In line with the workflow patterns being descriptive with an aim of completeness describing all used approaches rather than prescriptive we support neither all workflow control-flow patterns nor all workflow data patterns.

The possibility of business processes spanning multiple parties, i.e. collaborative business processes enables competition and efficiencies of jointly achieving business goals (Kutvonen et al., 2005). Based on this premise Kutvonen et al. explore how cloud-based BPM can be useful in supporting those collaborative enterprise needs.

In general, collaborative business processes involve many partners, activities and data. Different activities are naturally executed by different partners. Data related to different activities is often seen as sensitive by its owners. As such it is not desirable to expose much data to other partners in the collaborative business process. Only agreed data and messages should be allowed to be passed to the agreed partners. This implies that data and the execution of activities must be distributed amongst the partners in the collaborative business process. The collaborative process model does however require the coordination of different activities as they are executed by different partners towards a common goal. The collaborative process itself could be shared among the partners even if the data and activity implementation is kept confidential.

The case of industrial incident and maintenance management in a modern regulated energy market as presented in Section 2 exposes the issues of data confidentiality and robustness well. The case exposes fast change and dynamic processes. While it does have relevance to the case, resource elasticity of cloud-enabled business process management is out of scope for this paper.

Section 3 presents an architecture meta-model that supports collaborative businesss processes with data and activity confidentiality and sharing restrictions. This model is used in Section 4 as the basis of the architecture for a cloud based incident notifcation system. The service improvment of such a system is analysed in Section 5 . Section 6 evaluates the performance. A review of related work in Section 7, leading to the conclusion in Section 8.

\section{Motivating Case}

The case for BPaaS is well-illustrated through the example of incident and maintenance management in the Spanish electricity system. This system is typical of modern liberalized electricity systems with complex relationships among parties may simultaneously be collaborators as well as competitors. In addition to this complex relationship, there is a non-trivial regulatory framework that allows competition in this traditionally monopolistic market.

The Spanish electricity system is formed of a high-voltage electric power transmission network and grid connecting power stations and substations to transport electricity from where it is generated to where it is needed. Electricity transmission starts from power stations where energy is generated from various sources by generators. The generated electricity is then transformed for transport over the nationwide high voltage transport network (up to $400 \mathrm{kV}$ ). When the electricity reaches transformer substations (owned by the various distributors), the voltage is reduced to a local distribution level $(132 \mathrm{kV})$. This voltage is then supplied to the largest industrial users as well as to distribution substations. There the voltage is reduced to a medium level $(12.5 \mathrm{kV})$ for local distribution, either as end-product to larger industrial customers or through neighbourhood transformers for consumers and small businesses.

There are a number of stakeholders in the Spanish electricity system, each fulfilling various roles in the overall process of electricity generation and delivery. Many of the well-established, former government, parties play many of the roles in the system. The unavoidably monopolistic and critical roles of market 
operator (OMEL) and system operator (REE) remain in government hands. Other roles in the system are energy producers, carriers, distributors, marketers, and consumers.

\subsection{Incident Notification Concerns in the Spanish Electricity System}

The process of delivering electricity to a single customer involves an entire chain of roles (and therefore actors). The delivery of electricity to a single geographic group (for example, a street) of customers likely involves many more marketers. Given that most distributors, which have the function of distributing electrical energy, are also active as marketers on a national level these distributors generally act both as each other's collaborators/customers as well as competitors.

Incidents in the electricity system can occur anywhere and anytime. These incidents, ranging from signal errors, cabling problems to serious substation overloads, will affect energy supply, lead to power cuts or even generate a further huge impact to the community and economy.

Handling and resolving incidents requires coordination throughout the electricity system. As result of an incident on July 23rd, 2007 at the Collblanch substation in Spain, around 320,000 people were affected by energy cuts between 6 to 60 hours long (Press, 2013). A lack of information for the population resulted in disturbances on Barcelona's streets. After this incident, it became clear that effective incident management includes effective and timely informing of customers without relying on suddenly overloaded call-centres. The information provided should not only acknowledge the existence of an issue but also provide information on progress and estimated resolution timelines. When appropriate, follow-up notifications should be given to all or interested customers.

\section{Architecture Meta-model}

To aid in the description and analysis of the use of cloud based business process management in collaborative cases we present an architecture meta model. This architecture meta-model describes which object types are relevance, and which relations may exist among them, i.e. which partners are involved in which activities in which process models and handles which data, etc.

A service-oriented collaborative business process deployment is a specific deployed service with its configuration parameters. A deployed collaborative process model $p m \in P M$ is defined as a BPMN model with additional deployment data:

\section{Definition 1 (Process model components)}

Process models consist of a number of related components. The sets of these components are:

Ac

$E v \subseteq A c$

$\mathrm{Fl}$

Gw

St

En

$N i=A c \cup G w$

$N d=N i \cup S t \cup E n$

$\mathrm{Pa}$

$D t$

$D d=\mathcal{P}(D t)$

dataTypes: $A c \rightarrow \mathcal{P}(D t)$ Retrieve the data annotations

The set of possible activities

The set of possible events

The set of possible flows between process nodes

The set of possible gateways The set of possible start nodes The set of possible end nodes The set of inner process nodes The set of all process nodes The set of possible partners The set of possible data types The set of possible activity data anotations from an activity

Definition 2 (Gateway types) There are different types of gateways, either split (Sp) or join (Jo), in form of "and" $\left(S p_{\text {and }}, J_{o_{\text {and }}}\right)$, "or" $\left(S p_{\text {or }}, J o_{o r}\right)$, and "xor"(exclusive or) (Sp $\left.p_{x o r}, J_{o_{x o r}}\right)$.

$$
G w=S p_{\text {and }} \cup S p_{\text {xor }} \cup S p_{\text {or }} \cup J_{o_{\text {and }}} \cup J_{o_{\text {xor }}} \cup J_{o_{\text {or }}}
$$

Table 1 Activity Specifications

Activities

\begin{tabular}{|llc|}
\hline Activity 1: & Receive Alarm & REE \\
\hline Activity 2: & Received Affected Information & Distributors \\
\hline Activity 3: & Notifying Distributors & REE \\
\hline Activity 4: & Receive Incident Information by Marketers & Marketers \\
\hline Activity 5: & Receive Incident Information by OMEL & OMEL \\
\hline Activity 6: & Receive Incident Information by REE & REE \\
\hline Activity 7: & Monitoring disruption and publish information & Distributors \\
\hline Activity 8: & Publish Information & Marketers \\
\hline Activity 9: & Receive Confirmation of Notification & OMEL \\
\hline Activity 10: & Receive Confirmation of Notification & REE \\
\hline Activity 11: & Confirming Incident Over & Distributors \\
\hline Activity 12: & Receive Confirmation of Notification & Distributors \\
\hline
\end{tabular}


Definition 3 (Process models) Given the previously defined sets, the following operations on process models can be defined:

- flows : Pm $\rightarrow \mathcal{P}(F l)$ presents the process flows in a process model $\left(F l_{P m}\right)$;

- innerNodes $: \mathrm{Pm} \rightarrow \mathcal{P}(\mathrm{Ni})$ denotes the inner nodes of a process model $\left(N i_{P m}\right)$; and

- terminals $: P m \rightarrow \mathcal{P}(S t \cup$ En) show the start and end nodes in a process model.

Process models represent business processes and include possible activities and gateways; the start and end nodes in a process model; and flows between process activities and gateways.

Definition 4 (Activities and Partners) Activities $(\mathrm{Ac})$ are executed by a single partner $(\mathrm{Pa})$. Partners can execute multiple activities within a process. In addition, partner can involve in multiple processes. An activity is executed by one partner.

All activities in any model that a specific partner is involved in is defined through the relation $p a \rightarrow \mathcal{P}(A c)$.

The activities: $\mathrm{Pm} \times \mathrm{Pa} \rightarrow \mathcal{P}(A c) \quad$ relation represents the activities within a specific process model that a partner is involved in, i.e.

$$
\operatorname{activities}(p m, p a)=A c_{p a} \cap \operatorname{In}_{p m}
$$

partner: $A c \rightarrow P a$ is the partner that executes a particular activity.

There is a reflective relationship between partners and activities:

$$
\text { pa }=\operatorname{partner}(a c) \leftrightarrow a c \in \operatorname{activities}(p a)
$$

Definition 4 delineates the relationships between partners, activities and process models. The definitions are sufficient to determine which partners execute which activities in which process models. Table 1 shows what the values are in the motivation case (Section 2). Each row represents an activity, a description, and the performer of the activity. For example, REE performs activities 1, 3, 6, and 10 .

Figure 2 shows the process model at the basis for Table 1. The figure provides a detailed specification of the activities including which partners perform which activities. For example, the first row of the Table 1 represents Activity 1 is 'Receive Alarm' which is performed by REE. In the implementation of the case, the relationship between activites and partners is also represented as matrix $X$ in Section 6 .

Definition 5 (Data types and owners) $A$ partner performs an activity. An activity may associate with data. Data has its owners. Following equations identify the partner that performs an activity; the partners that process a datatype and any data should have at least one owner; and the activities that a data type is involved with respectively.

$$
\begin{aligned}
& \text { executor: } A c \rightarrow P a \\
& \text { accessors: } D t \rightarrow \mathcal{P}(P a) \backslash \emptyset \\
& \text { activities: } D t \rightarrow \mathcal{P}(A c) \\
& \text { activities }(d t)=\{a c \mid d t \in \operatorname{dataTypes}(a c)\}
\end{aligned}
$$

Table 2 presents a data perspective of the case detailing ownership and which activitie each data element is involved in. For example, row 1 of the Table 2 means that Data1 represents 'Database of distributor stations and substations', REE is the owner of the database.

In the implementation of the case, the relationship between data and partners (i.e. the owners of data) is represented as matrix $Q$ and the relationship between activities and data involved in different activities is noted as matrix $R$ in Section 6 respectively.

Table 2 further indicates relationship between data and activities; i.e. different data is used by different activities. For example, row 1 of Table 2 denotes that Data1 'Database of distributor stations and substations' is used by Activity 1. Row 5 describes Data 5 'BDT database' is used by both Activities 7 and 8 .

In the implementation of the case, the relationship between activities and data involved in different activities is noted as matrix $R$ in Section 6 .

Definition 6 (Valid models) Models are valid in terms of ownership if all activities are valid in terms of ownership. Activities are valid if activity executing partners have valid access to all the data needed for the activity.

$$
\begin{aligned}
\text { ownerValid }(a c)= & \forall_{d t}[d t \in \text { datatypes }(\text { ac }) \\
& \rightarrow(\text { executor }(a c) \in \operatorname{accessors}(d t))] \\
\operatorname{ownerValid}(p m)= & \forall_{\text {ac } \in \text { activities }(p m)}[\operatorname{ownerValid}(a c)]
\end{aligned}
$$

Algorithms 2 and 1 provide concrete implementations of this validation mechanism.

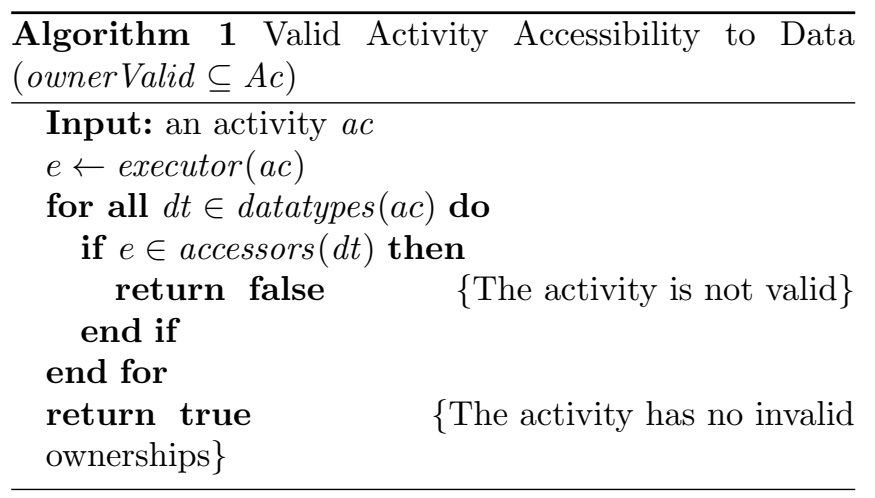

The above definitions allow us to reason about activities in a collaborative process as well as later to analyse the performance of the solutions, e.g. given a collaborative process model $\mathrm{pm}$

- which partners $p a \in P a_{p m}$ execute which activities $a c \in A c_{p m}$ in a given process model $p m$ ( activities: $\mathrm{Pm} \times \mathrm{Pa} \rightarrow \mathcal{P}(A c)$ )? 
Table 2 Data Item Specification

\begin{tabular}{|l|l|l|l|}
\multicolumn{2}{c}{ Data } & \multicolumn{1}{c}{ Description of Data } & \multicolumn{1}{c}{ Used by } \\
\hline Data 1: & Database of distributor stations and substations & REE & Activity 1 \\
\hline Data 2: & IDs/names of distributor station/substations & REE & Activity 1 \\
\hline Data 3: & Alarm information & REE & Activity 3 \\
\hline Data 4: & Detailed alarm information & REE & Activity 2 \\
\hline Data 5: & BDI database & Distributors & $\begin{array}{l}\text { Activity } 7 \\
\text { Activity } 8\end{array}$ \\
\hline Data 6: & A list of transformer centers & Distributors & Activity 7 \\
\hline Data 7: & SGC database & Distributors & $\begin{array}{l}\text { Activity } 7 \\
\text { Activity } 8\end{array}$ \\
\hline Data 8: & A list of affected streets and marketers & Distributors & Activity 7 \\
\hline Data 9: & Confirmation of incident over & Distributors & Activity 11 \\
\hline Data 10: & Incident detailed notification & REE & Activity 6 \\
\hline Data 11: & Incident information to marketers & OMEL & Activity 5 \\
\hline Data 12: & Confirmation of publishing incident information & Marketer & Activity 9 \\
\hline Data 13: & Confirmation of publishing incident information & OMEL & Activity 10 \\
\hline Data 14: & Confirmation of publishing incident information & REE & Activity 12 \\
\hline
\end{tabular}

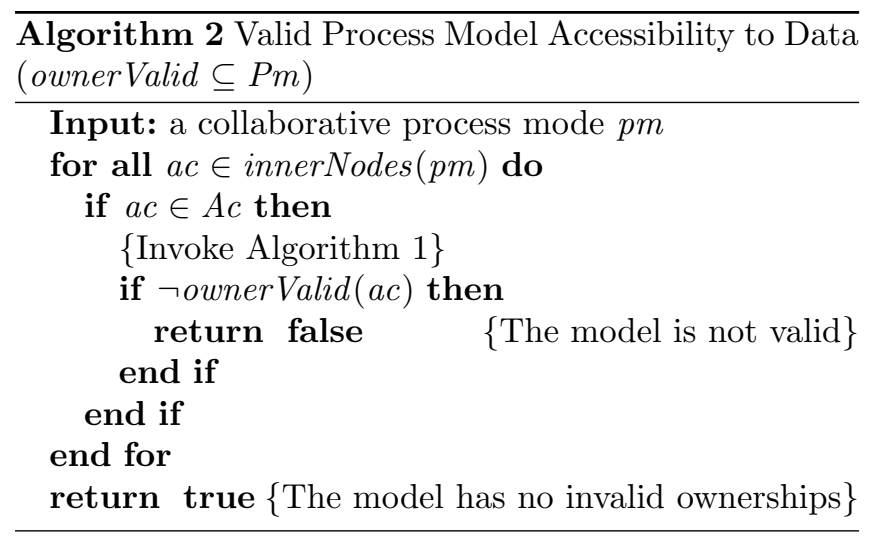

- which data items are required when activity $a c \in$ $A c_{p m}$ is executed in a given collaborative process model pm ( datatypes: $A c \rightarrow \mathcal{P}(D t))$ ?

Both incidents and maintenance can occur in the electricity grid, transformer stations, and/or transformer substations. The different collaborative process models which relate to different situations are stored in a collaborative process model repository. End users select a suitable model $p m \in P M$, to deploy it. Examples of collaborative process models are managing incident processes (which occur in a transformer station), managing incident processes (which occur in a transformer substation), managing incident processes (which occur in the grid), notification processes of maintaining transformer stations, notification processes of maintaining transformer substations, notification processes for grid maintainance, etc. For each type process, there are configurable parameters to set for different situations, which particularly indicate which activities are executed by which partner with data.
The partner responsible for executing an activity is retrieved using the executor: $A c \rightarrow P a$ function. When the activity is to be executed this requires all data involved in this activity $a c \in A c$ to be available and have correct ownership as defined by the ownerValid relationship. The design of our implementation architecture, experiment, and performance evaluation are based on the architecture meta-model. The usage of the architecture meta-model is further described in Section 5 and Section 7.

\section{Implementation Architecture}

The architecture meta-model forms the basis of our cloud-based notification systems (CNS). The case of incident notification in the Spanish electricity system has a number of properties. The case involves various parties, some of which are direct competitors, or even fulfilling the roles of supplier, distributor and competitor concurrently (this set-up is similar in various other countries). For commercial reasons, proper separation between the systems must therefore be maintained.

The nature of incident management is such that incidents are (relatively) short-lived and all are unique. At the same time, the electricity system case shows that high speed of execution (fast notification within minutes of occurrence) as well as reliability are still expected. In other words, automation is indicated but flexibility and cross-organisational capabilities should be included.

To meet the flexibility requirements in our proposed solution to the case, we use our previously discussed solution of business process mashup engines (de Vrieze et al., 2011) and lightweight business process modelling technology (Xu et al., 2010; Xie et al., 2010). 

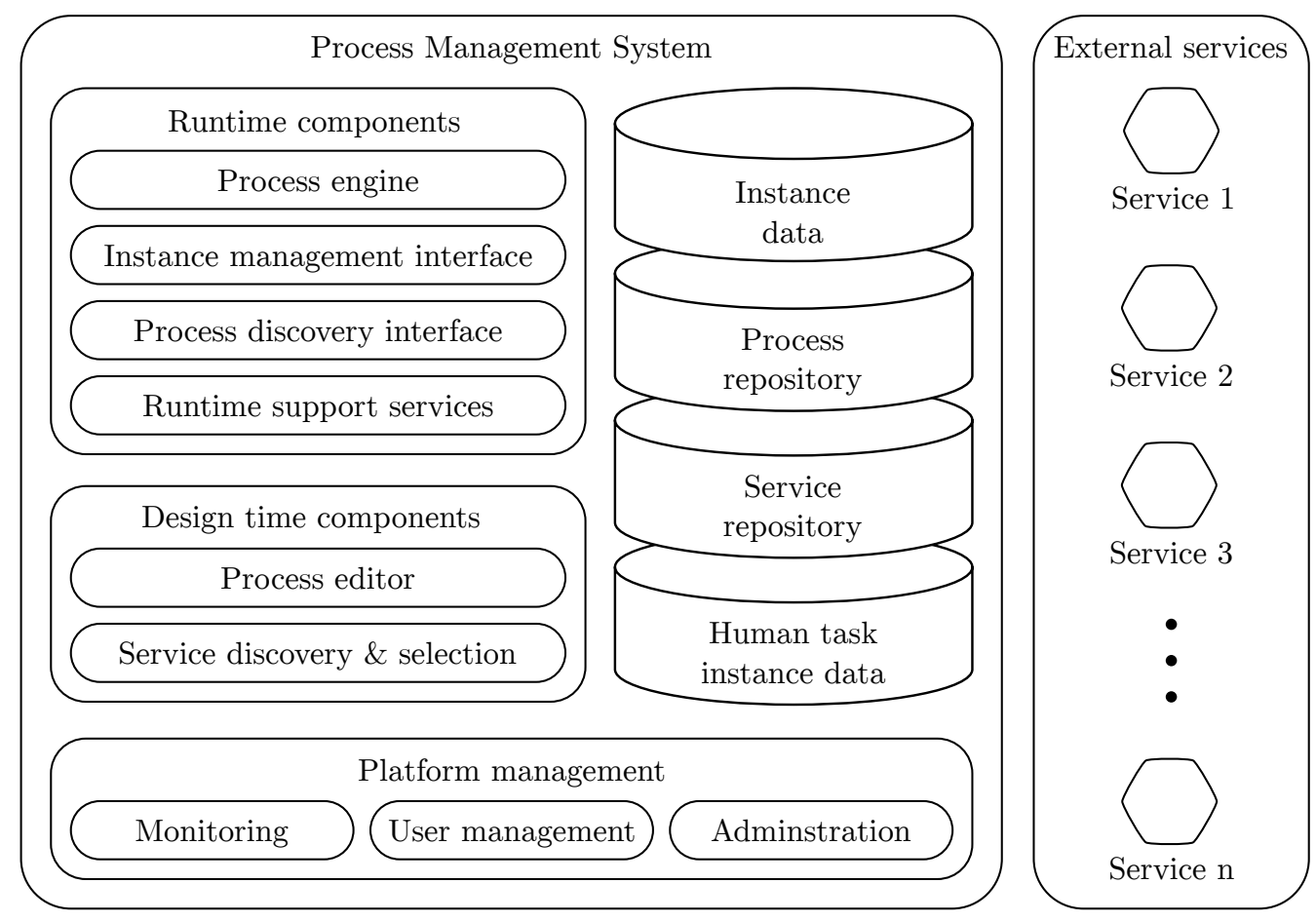

Figure 1 Architecture of the Process Mananagement system

These process-oriented mashup engines are deployed for all stakeholders of the Spanish electricity system, which includes OMEL, REE, distributors, transformer station, transformer substations, and marketers. This insures that all involved stakeholders can flexibly deal with incidents. This generic system could also be used for other applications, such as interruptions or regular maintenance within transformer or distribution substations.

In the case of incident notification the collaboration requirements in the process are limited in that there is a unidirectional flow in the process (there are no crossorganisational joins). As such this case does not require full support for fully federated business processes.

Figure 1 presents the architecture of the process management system that underpins the notification system. This figure contains both the process management system as well as external services. These services provide the implementation of actual business functionality beyond process management.

The process management system itself consists of three categories of active components as well as various datastores. The system itself is highly service oriented, and different roles are separated into different (decoupled) interfaces. The platform management modules provide various functionality that is used in the administration of the system. Initial modules would be the management of users (possibly through a corporate identity system such as active directory), administration of the process management system through linking capabilities to roles etc. Finally the monitoring of the system on a centralised level is important, both from a resource perspective as from the perspective of governance.

The design time components focus on the functionality needed to create or edit processes. Part of this is a subsystem that provides for the discovery and selection of web services as stored in a process repository. This subsystem would find candidate processes while taking the context (such as the current process model, as well as the user's role and past preferences) into account to reduce the friction in service selection. The process editor provides for the actual editing and creation of process models, often by copying and adapting existing models as well as pre-existing building blocks.

A variety of run-time components is needed for the execution of processes. Primary is the process engine. This engine is responsible for composing the invocation of the web services that make up the activity execution. It is also responsible to maintain the actual state of process instances and their associated data. It is however unaware of the specifics of web services as that is specified largely in the process models itself, or through the services used.

There are a number of runtime support services relevant to the system, primarily for the interaction with users through tasks. This is implemented through the concept of a process aware web service that, instead of responding only on completion, responds with a state update (and callback information) such that the process engine and service can update each other on task state (activity instances that have not been moved to a taken state can still be freely cancelled, perhaps to satisfy split constraints, and cancellation would be acceptable until progression into started). 


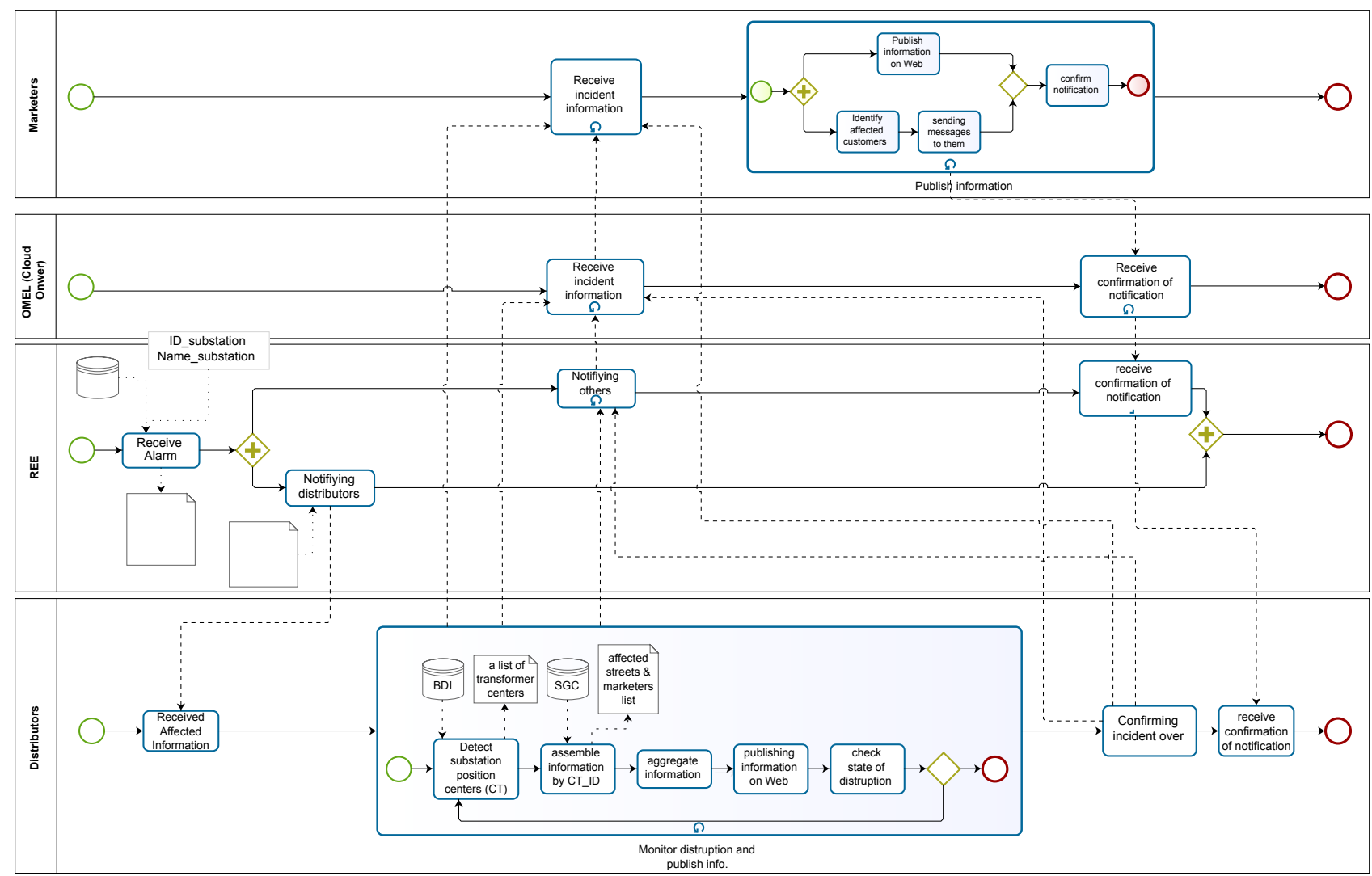

Figure 2 Cloud-based Notification Process Model

In cases where users themselves are responsible for their own processes, they need the ability to monitor and manage their process instances. This is performed by a process management interface that provides this functionality based upon primitive API's provided by the process engine. To allow users to find the processes they need, the process discovery interface provides a socially linked, context-aware system to find these processes.

After all data sources and invoked services are (semi) automatically indicated, process models are running as process instances at the runtime. The decentralised execution of process instances is a centre of the incident notification system in the incident notification system case. Although the information needed is not large in terms of data size (so transfer would not be a technical challenge) from both distributors and marketers, there are data sensitivity issues to face. While the information from the distributors or REE is not commercially sensitive, the data from the marketers is.

An example of a process model is presented in Figure 2, which could be used within the architecture. For the purpose of incident and maintenance notifications, different partners can use the notification systems in different ways.

Incidents or interruptions can be caused or observed in different parts of the distributors. Some sample process models can be modified according to the situation by the relevant parties. The incident notification process continually monitors the process of the repair. The process also ensures information is consistently published on the Web using the private process engine.

A common process model could be downloaded from the collaborative process model repository on the cloud by a marketer, who can further modify the models. For example to select different channels, such as sending Facebook messages and SMS messages for notifications. A local process editor can be used to allocate the data (affected customers' mobile phone number, Facebook ID, or Twitter ID). The selected model can be run on a private process-oriented mashup engine.

Maintaining the process model repository and on providing some common Web services are main concerns of the solution owner, i.e. OMEL, who provides a process editor and a process engine for users testing the process model cloud execution. The other users upload their data for running their activities when the private process engine is out of order. A collaborative process which runs in the cloud can be supported for special cases, e.g. monitoring the collaborative process.

Supporting regular maintenance notification is another usage of CNS. For example, regular maintenance can take place at transformer or distribution substations. National or local grid maintenance needs coordination with partners. Sometimes regular maintenance activities could also be a cause of an incident. Pre-designed collaborative process models are stored in the repository for different purposes for sharing and reuse. 


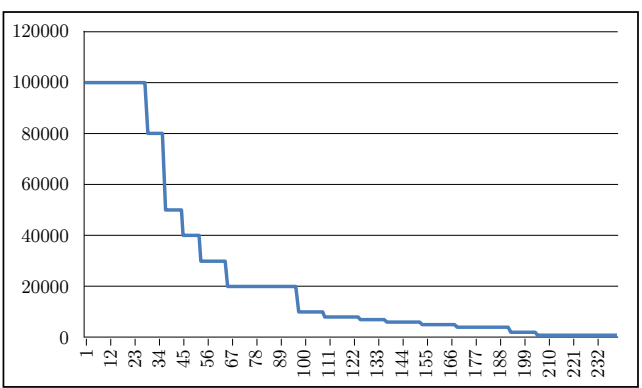

(a) Workload scenario 1

Figure 3 Hypothetic Workload Scenarios

\section{Service Improvement}

Looking back at the serious incident we mentioned in Section 2, we see that around where 320,000 people were affected. There were energy cuts of between 6 to 60 hours on July $23^{\text {rd }}$, 2007. A lack of information for the population resulted in disturbances on Barcelona's streets. The population's dissatisfaction was felt by the government and both companies implied: REE and ENDESA. The matter was brought to court: around 40 million Euros was demanded by the judge, and 21 million Euros by the Generalitat (Catalonia government). In addition, the National Energy Commission punished both companies. However, it is difficult to know the total amount of money spent on the incident and the impact concerning the customers' switch to other companies (Sanchez, 2007).

One way to improve notification service improvement is to increase staff members of call centres. Our paper (Xu et al., 2015, 2013b) provides detailed simulation results for different service level objectives. There are numbers of benefits for our solutions.

- the solution saves the need for extra personnel to handle extra calls when an incident starts;

- the solution provides a reliable notification process. Log files can be served as evidences for the future investigation of responsible partners when affected customers are not notified.

- the distributors do not have access to contact details of domestic customers, but nonetheless they can be held responsible for informing customers;

- the marketers can keep their sensitive customers' contact information.

Another way to see service improvement is look at the potential cost of handle an incident. We take the Barcelona case. 320,000 people roughly equates to 100,000 households. It takes less than 5 minutes to send 100,000 SMS messages out ${ }^{1}$. The cost of sending the messages depends on agreements between each partner with SMS providers but bulk packages do exist with significant discounts on retail prices. For analysis purposes, we make two assumptions. The

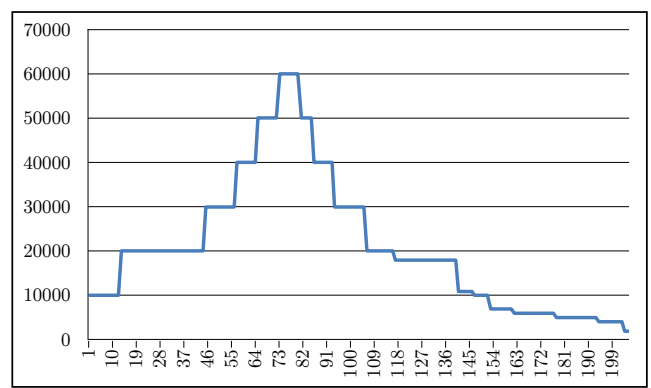

(b) Workload scenario 2

first assumption is the cost of sending SMS messages. The cost is assumed as 3.5 Eurocent per message. The messages related to the incident and updating information are sent out every a half hour. The second assumption is amount of SMS messages sent.

Based on these assumptions Figure 3 shows two hypothetical workload scenarios. The first workload scenario in Figure 3(a) shows an incident/maintenance event that initially impacts a large amount of households. For most households the issues are resolved early such that no further notifications are required. An example of such an incident could be a broken cable where neighbourhoods and other larger groupings of households are gradually rerouted.

The second workload scenario in Figure 3(b) depicts an incident/maintenance event that escalates before slowly being resolved. Initially few households are impacted, but this amount grows significantly before the issues are eventually resolved gradually. An example of such an incident could be cascading failures such as impacted the North East US in 2003 (Andersson et al., 2005 ) where the failure of one substation leads to related failures across the system.

The total amounts of messages sent are same for both workload scenarios in Figure 3(a) and (b), all 100,000 households will receive the messages during first six hours. Later on, only parts of 100,000 households will receive the related messages. After 60 hours, the incident was solved, no further messages need to be sent. In the end, a total of 5,826,000 messages has been sent for a total costs up to 203,910 Euro for Figure 3(a) and (b). There are 32 marketers in Spain ${ }^{2}$. Depending on how many customers of each marketer has the cost could be roughly 6,372 Euros for each marketer. Comparing to the fine of about 61 million Euros, it is a very economical and sustainable solution using CNS.

The notification process depends very much on the efforts of the marketers. There is no effective mechanism for monitoring the marketers' notification efforts. Customer satisfaction will finally determine and reinforce marketer behaviour. The marketers who did notify the customers well, will keep their customers while the marketers with less efforts may lose their customers.

Besides solving the notification problem, as a second benefit, incident or interruption notification process 
models can be shared among distributors, REE and marketers. An improved process model can be uploaded by each stakeholder and managed by the market operator OMEL. Third, the (Web) services related to the incident notification process can also be shared to improve performance. Multiple process mashup engines run at different sides, which also allow them to function as backups for each other. In general, a processoriented solution will be able to significantly reduce the communication time needed to provide adequate awareness of incident and related information among domestic and small business customers.

\section{Performance Evaluation}

To analyse the performance of the solution, we analyse the time involved in some potential scenarios. Our time cost model below is based on the time cost model in (Han et al., 2010). Han's time cost model focus on business processes within an organisation. In our research, we are dealing with multi partners involved collaborative process models, the new time cost model is thus extended to support multi-party collaboration.

Aligned with our definitions in Section 4, let us assume there is an activity $a c_{i} \in A c$. There are $k$ different collaborative partners $p a_{k} \in P a$ involved in the process. The execution time of activity $a c_{i}$ at the partner $p a_{k}$ is $t_{k}\left(a c_{i}\right)$. Let $X$ be the relation matrix of partners and activities, $X_{(k, i)}=1$ represents that partner $p a_{k}$ executes activity $a c_{i} ; X_{(k, i)}=0$ represents that partner $p a_{k}$ does not execute activity $a c_{i}$. The example of the matrix $\mathrm{X}$ for the collaborative process model in Figure 2 .

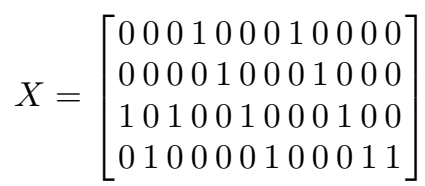

Each row represents different partners, each columm denotes different activities. For example, for row 4 i.e. $(0,1,0,0,0,0,1,0,0,0,1,1)$, distributors will process activities 'Received affected Information' (activity 2), 'Monitor disruption and publish info' (activity 7), 'Confirming incident over' (activity 11) and 'Receive confirmation of notification' (activity 12).

Let $b$ be reference bandwidth among involved partners in bytes per second. If a involves a set of data items (input and output) $D=\left\{d t_{1}, d t_{2}, \ldots, d t_{r}\right\}$ to be transferred among involved partners, then the transmission time needed is $\sum_{d t \in D} \frac{\text { size }(d t)}{b}$, where size is a function representing the size of data.

Let $Q$ be the relation matrix of partners and data. $q_{(k, j)}=1$ represents that data item $d t_{j}$ is located at the partner $p a_{k}$ side; $q_{(k, j)}=0$ means that data item $d t_{j}$ is not located at the partner $p a_{k}$ side. If $\forall k, \mid x_{(k, i)}-$ $q_{(k, j)} \mid=0$, it represents that activity $a c_{i}$ and data $d t_{j}$ are on the same side. The example of the marix $Q$ specifies the relation of data and its owner. Each row of the matrix expresses the owners of data and each coloum denotes which data the owners have. For example, row 3 (11110000010001) means that REE is the owner of Data 1 'Database of distributor stations and substations', Data 2 'IDs/names of sitributor stations/substations', Data 3 'Alarm information', Data 4 'Detailed alarm information', Data 10 'Incident detailed notification' and Data 14 'Confirmation of publishing incident information'.

$$
Q=\left[\begin{array}{llllllllllllll}
0 & 0 & 0 & 0 & 0 & 0 & 0 & 0 & 0 & 0 & 0 & 1 & 0 & 0 \\
0 & 0 & 0 & 0 & 0 & 0 & 0 & 0 & 0 & 0 & 1 & 0 & 1 & 0 \\
1 & 1 & 1 & 1 & 0 & 0 & 0 & 0 & 0 & 1 & 0 & 0 & 0 & 1 \\
0 & 0 & 0 & 0 & 1 & 1 & 1 & 1 & 1 & 0 & 0 & 0 & 0 & 0
\end{array}\right]
$$

Let $R$ be the relation matrix of activities and data, $r_{(i, j)}=1$ represents that activity $a c_{i}$ processes data $d t_{j} ; r_{(i, j)}=0$ represents that activity $a c_{i}$ does not processes data $d t_{j}$. An example of the matrix $R$, which shows which data will be involved in executing different activities in Figure 2. Each row denotes activities and each column indicates data, which used by different activities. For example, row 3 (00100000000000) represents that data 3 is used to perform Activity 3 'Notifying distributors'.

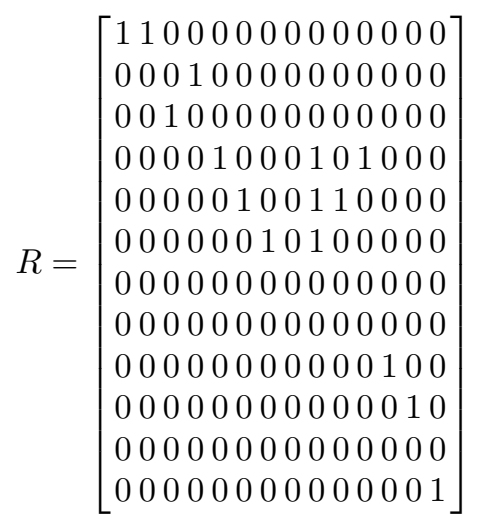

The time estimation model is followed:

$$
\begin{aligned}
T= & \sum_{k=1}^{o} \sum_{i=1}^{m} t_{k}\left(a c_{j}\right) X_{(k, i)} \\
& +\sum_{k=1}^{o} \sum_{i=1}^{m} \sum_{j=1}^{n} \frac{\operatorname{size}\left(d t_{j}\right)}{b} R_{(i, j)}\left|x_{(k, i)}-q_{(k, j)}\right|
\end{aligned}
$$

As provided case in Section 2, the process of sending incident notification to domestic consumers is a highly collaborative process. Each involved partner needs to provide data collaboratively. Involved partners are responsible for correction of their data. According to the process in Figure 2, besides all marketers, all participated organisations run their data individually based. The marketers can decide to send their customers' basic contact information to the cloud owner OMEL for distributing all notifications. Basic customers' information should not be extensive on individual basis, but may involve a large amount of 


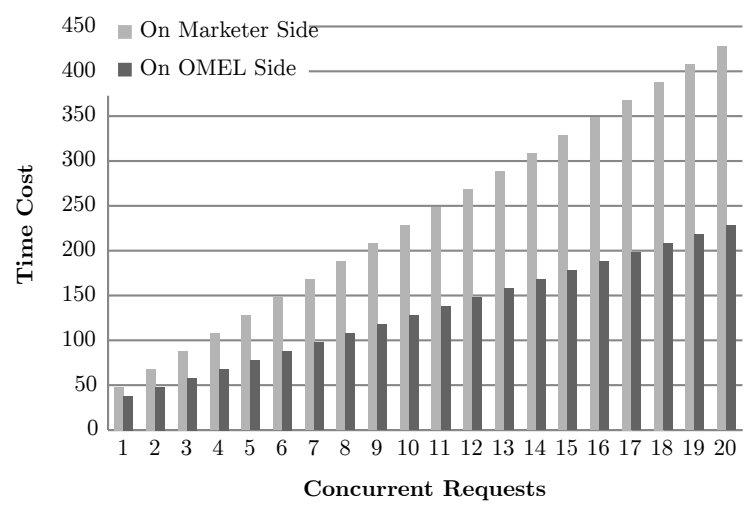

Figure 4 Time Cost Comparison

households. Alternatively, the marketers can also send incident notifications to their customers after the OMEL announcement.

To run the experiment, we distinguish two Internet network bandwidths: one is among involved enterprises; and another is between marketers and the cloud. We classify the execution time of activities into two kinds. The activities on the marketers' sides are processing a (relatively) large volume data where other activities in other organisations are not. We assume that the average size of customer data for each marketer is 300 bytes. The ASDL bandwidth is $3 \mathrm{Mbps}$. Therefore, the time to transfer data among different partners can be ignored. In Figure 4, we provide a comparison between processing notification in OMEL side and processing notification in individual marketers' sides. We also assume that the computing powers and capabilities available to the marketers (especially pure marketers) is generally lower than available in the cloud and to the other stakeholders. When the data is processed by the marketers, all data needs to be retrieved from CRM systems. Alternatively basic contact data such as address and telephone numbers could be provided to OMEL. It could then directly contact these customers. The speed of sending notification to domestic customers by OMEL is certainly faster than the speed of notifications being sent by individual marketers.

Figure 4 does not consider using elastic cloud resources. In Figure 5, we provide a comparison between two scenario workloads (see Figure 3 ) processing notifications in OMEL side. The OMEL side uses auto-scaling (Mao and Humphrey, 2011) to minimize computing cost in a cloud-based environment. In Figure 4 , the dashed line represents the needed computing power without auto-scaling virtual machines (VRs) and the solid line denotes the computing power with auto-scaling VRs involved. In this experiment, the scaling policies are set as: if the average CPU load is below $40 \%$ for five minutes, the number of virtual machine is reduced; if the average CPU load is above $70 \%$ for five minutes, one new virtual machine is added (Janiesch et al., 2014). The speed of sending notifications to domestic customers by OMEL is even faster. Especially for the more intensive and multiple incidences/maintenances running at the same time, sending notifications from the OMEL side shows the advantage of elastic computing power.

\section{Related work}

The problem of BPaaS support for collaborative business processes can be analysed from a number of perspectives. The first perspective considers collaborative business management, leading to the second perspective, cloud solutions for incident management. The following subsections in turn discuss related work in each of these areas.

\subsection{Cloud-based BPM and Collaborative Business Process Management}

A good amount of work on providing cloud-based business process management has appeared in recent years. It specially attracts small and medium enterprises. The business process management services can be used as a pay-per use manner (Buyya et al., 2008), which provide the enterprises scalability and lower maintenance costs. Here are some of major BPM services on the cloud: Amazon EC2 cloud (Amazon, 2013), IBM's Blue Works (IBM, 2013), Ramco OnDemand ERP (Scheer and Habermann, 2000; Appandairajan et al., 2012), and Salesforce's sales cloud and market cloud (Salesforce, 2012). These services are at different cloud levels, i.e. IaaS BPM, PaaS BPM, and SaaS BPM respectively.

By the nature of our research problem, the research presented in this paper does not deal with demand of computing powers or resources. In fact, our motivating case does not require large data analysis or access large volume resources, i.e. strong computing power is not needed. Elastic processes (Stefan et al., 2015) are subject to change the infrastructure in which computing storage and network element as well as applications are added or removed on-demand. One of infrastructural challenges for elastic processes is decentralise coordination for process enactment, which is limited to only see 'process enactment' phase. Our research however covers the process modelling phase, i.e. supporting common access to a process repository. The architecture supporting elastic business process management systems perform process deployment, i.e. deploy executed business process into different virtual machines which is not our research in this paper.

Montarnal et al. (2014) proposed a business process collaboration platform that supports discovery of potential collaboration partners based upon provided collaboration goals. In addition it facilitates building collective business process models based upon the capabilities of the discovered partners. This is significantly different from the goals of our research in that the pool of potential collaborators is not restricted. In our case there is a defined set of collaborators, 

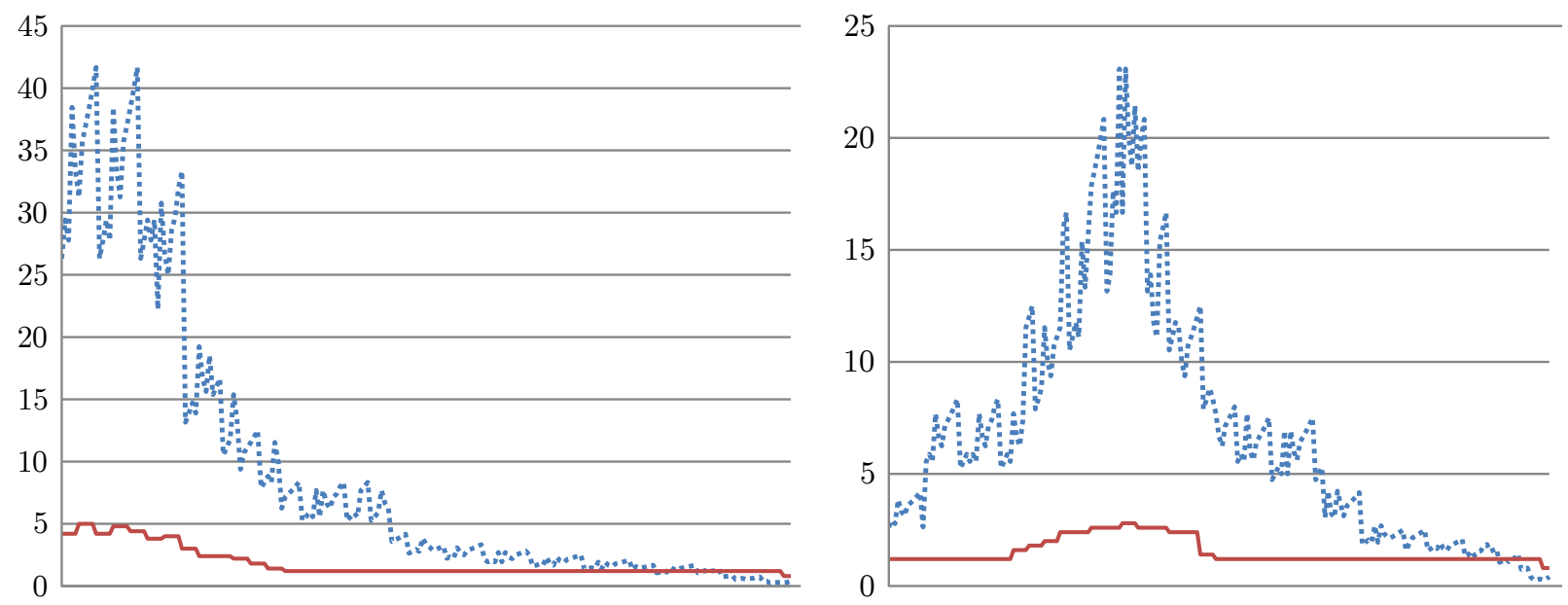

Figure 5 Computing Power Costs of Two Hypothetic Workload Scenarios

which however can be made to accommodate changes such as to enable the (mandated) collaboration. As such, our work does not concern the identification of collaboration partners, and neither are those partners restricted to their current offerings. The collaboration objective is static, as such automated generation of models, with the accompanying errors due to incomplete specification, based upon it is not needed either. Our research focuses on how to support end users of the Spanish electricity system in selecting some existing collaborative processes from process repository. The end users can further modify their own (linked) copies according to their needs. By the nature of incident management, we have also paid attention to reliability of the system. Therefore, unlike what was proposed in paper (Montarnal et al., 2014) distributed process engines are deployed around the collaborative partners to allow failovers and eliminate single points of failure, which is also different with the proposed approach.

NetMES system as a distributed manufacturing environment was proposed and implemented in (Helo et al., 2014). NetMES, a PaaS solution, provides information sharing and transferring in the manufacturing execution platform. In order to support monitoring, information exchange and other real-time interactions, cloud technology and service oriented technology are adopted. Preliminary only information and messages are exchanged among partners in the manufacturing executing system. In this paper, we proposed a BPaaS solution which goes further; it does not only treat the cloud as a platform of exchange information. The proposed cloud-based notification system allows sharing collaborative business process models. Data and activities distributed in cloud could be executed among participating parties according to the selected collaborative business process model.

A cloud-based solution for a television broadcast company is presented in the papers (Santos and Pires, 2012; Duipmans et al., 2014). The company allows its audiences to submit their program ideas, which could contain users' information, text description and a short video of their ideas. The computation-intensive activities, such as conversion and analysis of the video, are currently executed in the cloud. Video storages are resource consuming, without using a cloud solution, it requires further investment to original asset management system. The original business processes within a single company are thus move to the cloud and the involved processes are thus changed into collaborative processes between local systems and cloud solutions (computing resources and storage). The benefit of choosing cloudbased solutions for the television broadcast company is mainly that the company can benefit from the flexibility of cloud computing resources to meet peak demand and the unlimited storage space without further investing in in-house resources. First, our proposed solution is not for a single organisation. It supports multi-partners involved collaborative business processes in cloud environment. Secondly, a shared process executes collaboratively in the cloud environment. More significantly the proposed solution supports the changes of processes, ie. selecting, editing, and executing collaborative process models. Above mentioned three points makes the significant difference from our work to their work.

A decentralised collaborative process management system is presented in (Chen and Hsu, 2001). In this approach, collaborative process execution is based on a business process model and the design of an inter-enterprise collaborative business process management integrates using E-Carry with E-Speak. The potential collaboration among partners is hard code in advance. Our solution supports distributed collaborative processes in a flexible way, i.e potential collaboration can be formed selecting collaborative process models from process repositories and running them by process-oriented mashup engines. Although the idea of using collaborative process model to support business collaboration is common for both solutions, how to support business collaboration in a flexible way is different. 


\subsection{Cloud-based Solutions for Incident Management}

Cloud-based notification is built upon our previous research, namely business process mashup engines, lightweight business process modelling technology, earlier solutions and related analysis of the solutions. The design and implementation of a business process mashup engine is introduced in (de Vrieze et al., 2011), while the process engines are installed over the cloud. Lightweight business process modelling principle and modelling language (Xu et al., 2010; Xie et al., 2010) are used to specify the process modelling environment. Special attentions of our research are paid to the enduser aspect which is orthogonal to extending our designs for cloud-based business process management. A data as a service solution for the same case is discussed in paper (Jiang et al., 2012), which is a data central solution. Papers (Xu et al., 2013b, 2015) presents our earlier work for implementing cloud-based notification system, which service improvement are analysed. Resilience of the proposed solution is another important aspect of such system, which is analysed in (de Vrieze and $\mathrm{Xu}$, 2015). Significant improvement of this work, we provide an architecture meta-model as theoretical bases of our design and performance analysis. Further analysis of our solution and a detailed performance evaluation are provided in this paper.

\section{Conclusion}

BPaaS brings many benefits to business users. A cloudbased BPM solution provides a good opportunity, not only for small and medium enterprises, but also for large organisations which seek scalable and flexible solutions. Business process models are assets for many enterprises. To achieve business agility, business process collaboration needs to seamlessly connect local BPM systems and cloud-based BPM systems. The supported platform should also link to distributed data and activities according to business collaborations. This research is concerned how to support situational based flexible and agile collaborative process within a cloudbased environment. The proposed solution supports flexibility of business collaborations by selecting, editing, and executing the collaborative business process models. Lightweight business process modelling technologies, process-oriented mashup engines, and a process repository are foundations of the proposed solution of BPaaS.

The architecture meta-model is defined for supporting collaborative business processes as a theoretical foundation of the proposed solution. Lightweight business process modelling technologies (Xu et al., 2010; Xie et al., 2010), lightweight business process mashup engines (de Vrieze et al., 2011), and large collections of process models (Raduescu et al., 2006; Dijkman et al., 2012) are used to facilitate the solution of the incident notification processes to verify our design.

The core concept of the solution is that incident notification process models are verified and processoriented mashup engines to help stakeholders respond to emerging situations triggered by incidents more intuitively. The architecture supports on-demand and flexible situational applications which are used to address timely and immediate customer needs. We would like point out the existing SaaS ERP solutions e.g. RAMCO OnDemand ERP cannot allow instantly modify process models of different incident or maintenance notifications to fit end users' needs. Therefore, our CNS provides a solution in flexible collaborative business environment that these solutions do not offer.

Our cloud-based notification system can reduce the negative impact on the community and economy after an industrial incident occurs. From the end-user perspective, an effective incident management relies on instant situation awareness and response.

The case study has demonstrated how BPaaSs for collaborative processes facilitate in solving a real world problem effectively. Efficiency of the solution is analysed. The cost benefit of the solution has also been presented. Business process-oriented mashup engines are deployed in most of stakeholders which also improves reliability of the system(de Vrieze and $\mathrm{Xu}, 2015)$. Whilst the implementation was based on the Spanish electricity supply system, it can also be applied to other national electricity supply systems especially for the European Union countries in compliance with the EU Commission response on the applicability of art 17 of Regulation $2003 / 1$ to the gas and electricity sectors (Commission of the European Communities, 2007).

Virtual factory supports integrating ICT technologies and collaborative applications into traditional manufacturing practices and processes. This allows for increased flexibility in manufacturing, mass customization, increased speed, better quality and improved productivity. The emergence of service process modelling describes precisely the composition of manufacturing and related services. Papers (LatorreBiel et al., 2017),(Bazoun et al., 2016) provides different technology to simulate discrete event systems. Our future work will focus on extend the business process models to construct models of models of discrete event systems for the purpose of DEVS distributed simulation.

\section{Acknowledgements}

This research has been partially sponsored by EU H2020 FIRST project, Grant No. 734599, FIRST: vF Interoperation suppoRting buSiness innovaTion. The authors would like to thank Mr. Oscar Jarabo for his early work and supports during the research. 


\section{References}

Rafael Accorsi. Business process as a service: Chances for remote auditing. In Computer Software and Applications Conference Workshops (COMPSACW), 2011 IEEE 35th Annual, pages 398-403. IEEE, 2011.

Allan Afuah. Dynamic boundaries of the firm: are firms better off being vertically integrated in the face of a technological change? Academy of Management journal, 44(6):1211-1228, 2001.

Amazon. Amazon elastic compute cloud (amazon ec2), 2013.

G Andersson, P Donalek, R Farmer, N Hatziargyriou, I Kamwa, $\mathrm{P}$ Kundur, $\mathrm{N}$ Martins, J Paserba, P Pourbeik, J Sanchez-Gasca, et al. Causes of the 2003 major grid blackouts in north america and europe, and recommended means to improve system dynamic performance. Power Systems, IEEE Transactions on, 20(4):1922-1928, 2005.

P. Appandairajan, N.Z.A. Khan, and M. Madiajagan. Erp on cloud: Implementation strategies and challenges. In Cloud Computing Technologies, Applications and Management (ICCCTAM), 2012 International Conference on, pages 56-59, Dec 2012. doi: 10.1109/ICCCTAM.2012.6488071.

Hassan Bazoun, Judicael Ribault, Gregory Zacharewicz, Yves Ducq, and Hadrien Boyé. Slmtoolbox: Enterprise service process modelling and simulation by coupling devs and services workflow. International Journal of Simulation and Process Modelling, 11(6):453-467, 2016.

Mehdi Bentounsi, Salima Benbernou, and Mikhail J Atallah. Security-aware business process as a service by hiding provenance. Computer Standards \& Interfaces, 44:220-233, 2016.

Severine Blanc, Yves Ducq, and Bruno Vallespir. Evolution management towards interoperable supply chains using performance measurement. Computers in Industry, 58(7):720-732, 2007.

Pascal Bouvry. Emerging paradigms and areas for expansion. Cloud Computing, IEEE, 1(1):58-61, 2014.

Rajkumar Buyya, Chee Shin Yeo, and Srikumar Venugopal. Market-oriented cloud computing: Vision, hype, and reality for delivering it services as computing utilities. In High Performance Computing and Communications, 2008. HPCC'08. 10th IEEE International Conference on, pages 5-13. IEEE, 2008.

Qiming Chen and Meichun Hsu. Inter-enterprise collaborative business process management. In Data Engineering, 2001. Proceedings. 17th International Conference on, pages 253-260. IEEE, 2001.
Commission of the European Communities. Inquiry pursuant to article 17 of regulation (ec) no 1/2003 into the european gas and electricity sectors (final report) (com/2006/0851 final), 2007.

Paul de Vrieze and Lai Xu. An analysis of resilience of a cloud based incident notification process. In Risks and Resilience of Collaborative Networks, pages 110-121. Springer, 2015.

Paul de Vrieze, Lai Xu, Athman Bouguettaya, Jian Yang, and Jinjun Chen. Building enterprise mashups. Future Generation Comp. Syst., 27(5):637-642, 2011.

Remco M Dijkman, Marcello La Rosa, and Hajo A Reijers. Managing large collections of business process models-current techniques and challenges. Computers in Industry, 63(2):91-97, 2012.

Evert Ferdinand Duipmans, Luís Ferreira Pires, and Luiz Olavo Bonino da Silva Santos. A transformationbased approach to business process management in the cloud. Journal of grid computing, 12(2):191-219, 2014.

Gary Gereffi, John Humphrey, and Timothy Sturgeon. The governance of global value chains. Review of international political economy, 12(1):78-104, 2005.

Hongmei Gou, Biqing Huang, Wenhuang Liu, and Xiu Li. A framework for virtual enterprise operation management. Computers in Industry, 50(3):333-352, 2003.

Yan-Bo Han, Jun-Yi Sun, Gui-Ling Wang, and HouFu Li. A cloud-based bpm architecture with userend distribution of non-compute-intensive activities and sensitive data. Journal of Computer Science and Technology, 25(6):1157-1167, 2010.

Petri Helo, Mikko Suorsa, Yuqiuge Hao, and Pornthep Anussornnitisarn. Toward a cloud-based manufacturing execution system for distributed manufacturing. Computers in Industry, 65(4): 646-656, 2014.

IBM. Blueworkslive, 2013.

Christian Janiesch, Ingo Weber, Jorn Kuhlenkamp, and Michael Menzel. Optimizing the performance of automated business processes executed on virtualized infrastructure. In System Sciences (HICSS), 201447 th Hawaii International Conference on, pages 3818-3826. IEEE, 2014.

Nan Jiang, Lai Xu, Paul de Vrieze, Mian-Guan Lim, and Oscar Jarabo. A cloud based data integration framework. In PRO-VE, pages 177-185, 2012.

Riitta Katila and Paul Y Mang. Exploiting technological opportunities: the timing of collaborations. Research policy, 32(2):317-332, 2003. 
G Keller, M Nüttgens, and AW Scheer. Semantische prozessmodellierung auf der grundlage ereignisgesteuerter prozessketten (epk). 1992. Institut für Wirtschaftsinformatik, Universität des Saarlandes: Saarbrücken, 2009.

Kais Klai, Samir Tata, and Jörg Desel. Symbolic abstraction and deadlock-freeness verification of interenterprise processes. Data $\&$ Knowledge Engineering, 70(5):467-482, 2011.

Lea Kutvonen, Janne Metso, and Toni Ruokolainen. Inter-enterprise collaboration management in dynamic business networks. In On the Move to Meaningful Internet Systems 2005: CoopIS, DOA, and ODBASE, pages 593-611. Springer, 2005.

Lea Kutvonen, Toni Ruokolainen, and Janne Metso. Interoperability middleware for federated business services in web-pilarcos. Agent and Web Service Technologies in Virtual Enterprises, page 288, 2007.

Juan Ignacio Latorre-Biel, Mercedes Pérez De La Parte, and Emilio Jiménez-Macías. Enriching the formalism of coloured petri nets for modelling alternative structural configurations of a discrete event system: disjunctive cpn. International Journal of Simulation and Process Modelling, 12(1):92-101, 2017.

Dovev Lavie. The competitive advantage of interconnected firms: An extension of the resourcebased view. Academy of management review, 31(3): 638-658, 2006.

Frank Leymann, Dieter Roller, and M-T Schmidt. Web services and business process management. IBM systems Journal, 41(2):198-211, 2002.

Duen-Ren Liu and Minxin Shen. Workflow modeling for virtual processes: an order-preserving process-view approach. Information Systems, 28(6):505-532, 2003.

Ming Mao and Marty Humphrey. Auto-scaling to minimize cost and meet application deadlines in cloud workflows. In Proceedings of 2011 International Conference for High Performance Computing, Networking, Storage and Analysis, page 49. ACM, 2011.

Aurélie Montarnal, Anne-Marie Barthe-Delanoë, Frédérick Bénaben, Matthieu Lauras, and Jacques Lamothe. Towards automated business process deduction through a social and collaborative platform. In Collaborative Systems for Smart Networked Environments, pages 443-451. Springer, 2014.

Wenxin Mu, Frederick Benaben, Herve Pingaud, Nicolas Boissel-Dallier, and Jean-Pierre Lorré. A modeldriven bpm approach for soa mediation information system design in a collaborative context. In Services Computing (SCC), 2011 IEEE International Conference on, pages 747-748. IEEE, 2011.
John A Muckstadt, David H Murray, James A Rappold, and Dwight E Collins. Guidelines for collaborative supply chain system design and operation. Information systems frontiers, 3(4):427453, 2001.

Bjoern Niehaves and Ralf Plattfaut. Collaborative business process management: status quo and quo vadis. Business Process Management Journal, 17(3): 384-402, 2011.

Anil Nigam and Nathan S Caswell. Business artifacts: An approach to operational specification. IBM Systems Journal, 42(3):428-445, 2003.

Alex Norta and Paul Grefen. Discovering patterns for inter-organizational business process collaboration. International Journal of Cooperative Information Systems, 16(03n04):507-544, 2007.

OMG. Business process modeling notation (bpmn) 1.1., 2006 .

Michael P Papazoglou and Willem-Jan van den Heuvel. Blueprinting the cloud. IEEE Internet Computing, 15 (6):74-79, 2011.

Maja Pesic, Helen Schonenberg, and Wil MP Van der Aalst. Declare: Full support for loosely-structured processes. In Enterprise Distributed Object Computing Conference, 200\%. EDOC 200\%. 11th IEEE International, pages 287-287. IEEE, 2007.

The Associated Press. Barcelona blackout may last weeks, 2013.

Corina Raduescu, Cherri Tan, Malini Jayaganesh, Wasana Sedera, Michael zur Muehlen, and Sonia Lippe. A framework of issues in large process modeling projects. 2006.

Nick Russell, Arthur HM Ter Hofstede, David Edmond, and Wil MP van der Aalst. Workflow data patterns: Identification, representation and tool support. In International Conference on Conceptual Modeling, pages 353-368. Springer, 2005.

Nick Russell, Arthur HM Ter Hofstede, Wil MP Van Der Aalst, and Nataliya Mulyar. Workflow controlflow patterns: A revised view. BPM Center Report BPM-06-22, BPMcenter. org, pages 06-22, 2006.

Salesforce. Salesfource: Sales cloud, marketing cloud, 2012.

C. Sanchez. La cne prepara una sanción ejemplar contra endesa y red eléctrica por el apagón de barcelona, el confidencial, September 2007.

Luiz O Santos and Luis Ferreira Pires. Towards a bpm cloud architecture with data and activity distribution. In Enterprise Distributed Object Computing Conference Workshops (EDOCW), 2012 IEEE 16th International, pages 165-171. IEEE, 2012. 
August-Wilhelm Scheer and Frank Habermann. Enterprise resource planning: Making erp a success. Commun. ACM, 43(4):57-61, April 2000. ISSN 0001-0782. doi: 10.1145/332051.332073. URL http://doi .acm.org/10.1145/332051.332073.

Siew Kien Sia, Christina Soh, and Peter Weill. It governance in global enterprises: managing in asia. ICIS 2008 Proceedings, page 97, 2008.

Schulte Stefan, Christian Janiesch, Srikumar Venugopal, Ingo Weber, and Philipp Hoenisch. Elastic business process management: State of the art and open challenges for bpm in the cloud. Future Generation Computer Systems, 46:36-50, 2015.

Timothy J Sturgeon. Modular production networks: a new american model of industrial organization. Industrial and corporate change, 11(3):451-496, 2002.

Yutian Sun, Jianwen Su, and Jian Yang. Separating execution and data management: A key to businessprocess-as-a-service (bpaas). 8659:374-382, 2014.

Yutian Sun, Jianwen Su, and Jian Yang. Universal artifacts: a new approach to business process management (bpm) systems. ACM Transactions on Management Information Systems (TMIS), 7(1):3, 2016.

Wil MP van der Aalst. Structural characterizations of sound workflow nets. Computing Science Reports, 96 (23):18-22, 1996.

Wil MP Van Der Aalst, Arthur HM Ter Hofstede, and Mathias Weske. Business process management: A survey. In International conference on business process management, pages 1-12. Springer, 2003.

Wil MP Van Der Aalst, Lachlan Aldred, Marlon Dumas, and Arthur HM ter Hofstede. Design and implementation of the yawl system. In International Conference on Advanced Information Systems Engineering, pages 142-159. Springer, 2004.

Robert Woitsch and Wilfrid Utz. Business process as a service (bpaas). In Conference on e-Business, eServices and e-Society, pages 435-440. Springer, 2015.

Li Xie, Lai Xu, and Paul de Vrieze. Lightweight business process modelling. In E-Business and E-Government (ICEE), 2010 International Conference on, pages 183186. IEEE, 2010.

Lai Xu and Manfred A Jeusfeld. Pro-active monitoring of electronic contracts. In Advanced Information Systems Engineering, pages 584-600. Springer, 2003.

Lai Xu, Paul de Vrieze, Keith Phalp, Sheridan Jeary, and Peng Liang. Lightweight process modeling for virtual enterprise process collaboration. In PRO-VE, pages 501-508, 2010.
Lai Xu, Paul de Vrieze, Keith Phalp, Sherry Jeary, and Peng Liang. Interoperative end user process modelling for process collaborative manufacturing. International Journal of Computer Integrated Manufacturing, 26 (11):990-1002, 2013a.

Lai Xu, Paul de Vrieze, and Nan Jiang. Incident notification process as a service for electricity supply systems. In Proceedings of the 2013 IEEE Sixth International Conference on Cloud Computing, pages 926-933. IEEE Computer Society, 2013b.

Lai $\mathrm{Xu}$, Paul de Vrieze, and Nan Jiang. Incident notification process as bpaas for electricity supply system. International Journal of Cloud Computing, accepted, 2015.

Li Da Xu. Enterprise systems: state-of-the-art and future trends. Industrial Informatics, IEEE Transactions on, 7(4):630-640, 2011.

Dongjin Yu, Qi Zhu, Dalong Guo, Binbin Huang, and Jianwen Su. jbpm4s: A multi-tenant extension of jbpm to support bpaas. In Asia-Pacific Conference on Business Process Management, pages 43-56. Springer, 2015.

Liang-Jie Zhang and Qun Zhou. Ccoa: Cloud computing open architecture. In Web Services, 2009. ICWS 2009. IEEE International Conference on, pages 607-616. IEEE, 2009.

Michael zur Muehlen, Edward A Stohr, Jian Cai, Stephen CY Lu, François Grobler, Michael Case, and Nan Jing. Modeling and managing collaborative processes over the internet. Business process management journal, 11(3):255-274, 2005. 